\title{
Role of Preferential Crystallographic Orientation in Piezoresistance Anisotropy for Cubic Polycrystalline Aggregates
}

\author{
Taeko Ando ${ }^{*}$ and Toshiyuki Toriyama \\ Department of Mechanical Engineering, College of Science and Engineering, Ritsumeikan University, \\ 1-1-1 Noji-higashi, Kusatsu, Shiga 525-8577, Japan
}

(Received April 6, 2018; accepted August 3, 2018)

Keywords: piezoresistance, polycrystalline aggregates, cubic anisotropy, preferential crystallographic orientation, crystallite orientation distribution function

We derived an overall piezoresistance tensor for polycrystalline aggregates having an orthotropic texture using the crystallite orientation distribution function (CODF) and the cubic single-crystal piezoresistance tensor. We found that the deviatoric part of the overall piezoresistance tensor has a functional dependence on the preferential crystallographic orientation of cubic single crystals and that the tensor has three independent components. The physical properties of the tensor are invariant under the choice of the CODF. We applied the CODF defined by Roe to calculate the tensor for a fiber texture. Furthermore, we compared the experimental results for the piezoresistance of polysilicon with our theoretical analysis results. The experimental results indicate that the variation in texture strength plays a major role in representing the piezoresistance anisotropy of polycrystalline aggregates.

\section{Introduction}

Polycrystalline semiconductor materials are widely used as a force-sensing element of micro-electromechanical systems (MEMS). ${ }^{(1-5)}$ In the mid-1980s, French and Evans proposed a physical interpretation of the polycrystalline silicon (poly-Si) piezoresistance taking into account the ideal fiber texture. ${ }^{(6-8)}$ Since then, the number of published papers concerning piezoresistance in polycrystalline semiconductor materials has increased yearly. ${ }^{(1-15)}$ The huge amount of accumulated experimental data suggests that the piezoresistance in polycrystalline semiconductor materials strongly depends on the crystallite orientation distribution, i.e., the texture. $^{(8)}$ However, the physical role of the preferential crystallographic orientation in the overall piezoresistance anisotropy, i.e., the texture-induced piezoresistance anisotropy for polycrystalline aggregates, has not yet been established. Therefore, in this study, we derived an overall piezoresistance tensor for polycrystalline aggregates having an orthotropic texture in terms of the crystallite orientation distribution function (CODF) and the cubic singlecrystal piezoresistance tensor. Furthermore, as long as we define the texture distribution using appropriate spherical harmonics, the derived overall orthotropic piezoresistance tensor

${ }^{*}$ Corresponding author: e-mail: tando@fc.ritsumei.ac.jp

https://doi.org/10.18494/SAM.2018.1960 
is invariant for any choice of the functional form of the CODF. Thus, the obtained result is expected to be very useful for practical piezoresistance analysis for polycrystalline aggregates having cubic single crystals based on texture measurements using X-ray diffraction techniques. ${ }^{(16)}$

The organization of our paper is as follows. In the first part of the paper, we present the derivation of the overall piezoresistance tensor for polycrystalline aggregates having an orthotropic texture in terms of the CODF and the cubic single-crystal piezoresistance tensor. In the second part of the paper, we verify the consistency between the derived overall piezoresistance tensor and experimental results by the microfabrication and texture and piezoresistance measurements of a poly-Si piezoresistor.

\section{Derivation of Overall Piezoresistance Tensor for Polycrystalline Aggregates}

\subsection{Description of texture-induced piezoresistance anisotropy by means of CODF ${ }^{(17)}$}

The CODF defined by the three Euler angles $(\psi, \theta, \phi)$ shown in Fig. 1 can be used for a quantitative description of crystal texture. We assume that $V_{0}$ is the total volume of a polycrystalline aggregate occupied by all single crystal grains, and $\Delta V(\psi, \theta, \phi)$ is the partial volume occupied by single crystal grains contained within the element of orientation between $(\psi, \theta, \phi)$ and $(\psi+\Delta \psi, \theta+\Delta \theta, \phi+\Delta \phi)$ with respect to the material reference frame $O-x_{1} x_{2} x_{3}$. We can define the CODF as $W(\psi, \theta, \phi){ }^{(18)}$

$$
\frac{\Delta V(\psi, \theta, \phi)}{V_{0}}=W(\psi, \theta, \phi) \sin \theta d \theta d \psi d \phi
$$

If we perform an integration of the CODF with respect to the total volume of the polycrystalline aggregate, it is plausible from Eq. (1) that,

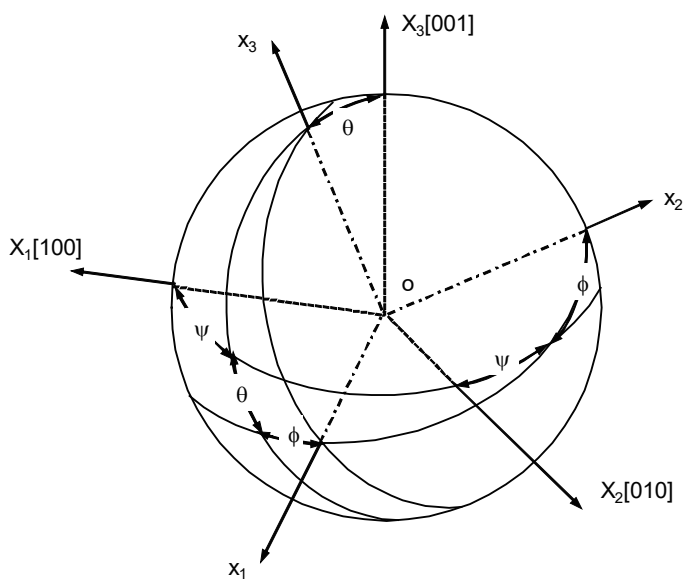

Fig. 1. Definition of Euler angle. 


$$
\int_{0}^{2 \pi} \int_{0}^{2 \pi} \int_{0}^{\pi} W(\psi, \theta, \phi) \sin \theta d \theta d \psi d \phi=\int_{0}^{2 \pi} \int_{0}^{2 \pi} \int_{-1}^{1} W(\xi, \psi, \phi) d \xi d \psi d \phi=1
$$

where $\xi \equiv \cos \theta$.

Then, the crystallographic orientation average of an arbitrary fourth-rank tensor $T_{i j k l}(\xi, \psi, \phi)$ can be calculated from Eqs. (1) and (2),

$$
\overline{T_{i j k l}}=\int_{0}^{2 \pi 2 \pi} \int_{0}^{\pi} \int_{-1}^{1} W(\xi, \psi, \phi) T_{i j k l}(\xi, \psi, \phi) d \xi d \psi d \phi
$$

We assume that a polycrystalline aggregate is composed of a huge number of cubic single crystals, which can be estimated as physical points, and that the stress applied to each crystal is the same as that applied to the polycrystalline aggregate or that the material is subjected to a remote average uniform stress. This averaging method is similar to the Reuss model for the elastic compliance tensor. ${ }^{(19)}$ We define two Cartesian coordinate systems corresponding to the material reference frame $O-x_{1} x_{2} x_{3}$ and the principal cubic single-crystal crystallographic frame $O-X_{1} X_{2} X_{3}$, as shown in Fig. 1. The orthogonal orientations of the two Cartesian coordinate systems can be related by three Euler angles $(\psi, \theta, \phi)$. If an index occurs twice in any term of the Cartesian tensor components, summation is taken from 1 to 3 . However, the capital letters (e.g., $L$ ) and Greek indices (e.g., $\alpha, \beta, \gamma)$ contained in the summation symbols (e.g., $\sum_{L=1}^{3} \sum_{\gamma=-\alpha}^{\alpha}$ ) obey the ordinary summation rule and the tensor summation convention does not apply to them. According to a previous work, ${ }^{(20,21)}$ a piezoresistance tensor having cubic anisotropy with respect to the crystallographic principal frame $O-x_{1} x_{2} x_{3}$ can be written as

$$
\Pi_{i j k l}=\pi_{1} \delta_{i j} \delta_{k l}+2 \pi_{2} I_{i j k l}+\pi_{3} \sum_{L=1}^{3} \delta_{i L} \delta_{j L} \delta_{k L} \delta_{l L}
$$

where $\delta_{i j}$ is the Kronecker delta, $I_{i j k l}=\frac{1}{2}\left(\delta_{i k} \delta_{j l}+\delta_{i l} \delta_{j k}\right), \pi_{1}=\Pi_{1122}=\Pi_{2211}=\Pi_{2233}=\Pi_{3322}=$ $\Pi_{3311}=\Pi_{1133}, \pi_{2}=\Pi_{2323}=\Pi_{3131}=\Pi_{1212}\left(\Pi_{2323}=\Pi_{2332}=\Pi_{3223}=\Pi_{3232}\right.$, and similarly for other components), and $\pi_{1}+2 \pi_{2}+\pi_{3}=\Pi_{1111}=\Pi_{2222}=\Pi_{3333}$.

The law of fourth-rank tensor transformation leads to the following piezoresistance tensor with respect to the material frame $O-x_{1} x_{2} x_{3}$ :

$$
\pi_{i j k l}=\Pi_{p q r s} \frac{\partial X_{p}}{\partial x_{i}} \frac{\partial X_{q}}{\partial x_{j}} \frac{\partial X_{r}}{\partial x_{k}} \frac{\partial X_{s}}{\partial x_{l}}
$$

where $\frac{\partial X_{p}}{\partial x_{i}}$ is the direction cosine between reference frames $O-X_{1} X_{2} X_{3}$ and $O-x_{1} x_{2} x_{3}$, and it can 
be expressed by a standard method using the three Euler angles $(\psi, \theta, \phi)$ shown in Table $1 .{ }^{(22)}$

Then, the crystallographic orientation average of $\overline{\pi_{i j k l}}$ can be written by using Eqs. (3) and (5) as

$$
\overline{\pi_{i j k l}}=\int_{0}^{2 \pi} \int_{0}^{2 \pi} \int_{-1}^{1} W(\xi, \psi, \phi) \Pi_{p q r s} \frac{\partial X_{p}}{\partial x_{i}} \frac{\partial X_{q}}{\partial x_{j}} \frac{\partial X_{r}}{\partial x_{k}} \frac{\partial X_{s}}{\partial x_{l}} d \xi d \psi d \phi
$$

where $\xi \equiv \cos \theta$ and $W(\xi, \psi, \phi)$ is the CODF described by the spherical harmonics. ${ }^{(23,24)}$

The substitution of Eqs. (4) and (5) into Eq. (6) leads to

$$
\begin{gathered}
\overline{\pi_{i j k l}}=\pi_{1} \delta_{i j} \delta_{k l}+2 \pi_{2} I_{i j k l}+\pi_{3} M_{i j k l}, \\
M_{i j k l}=\int_{0}^{2 \pi 2 \pi} \int_{0}^{1} \int_{-1}^{1} W(\xi, \psi, \phi) \sum_{L=1}^{3} \frac{\partial X_{L}}{\partial x_{i}} \frac{\partial X_{L}}{\partial x_{j}} \frac{\partial X_{L}}{\partial x_{k}} \frac{\partial X_{L}}{\partial x_{l}} d \xi d \psi d \phi \\
\equiv \int_{0}^{2 \pi} \int_{0}^{2 \pi} \int_{-1}^{1} W(\xi, \psi, \phi) A_{i j k l}(\xi, \psi, \phi) d \xi d \psi d \phi,
\end{gathered}
$$

where

$$
A_{i j k l}(\xi, \psi, \phi) \equiv \sum_{L=1}^{3} \frac{\partial X_{L}}{\partial x_{i}} \frac{\partial X_{L}}{\partial x_{j}} \frac{\partial X_{L}}{\partial x_{k}} \frac{\partial X_{L}}{\partial x_{l}}
$$

It is plausible from Eq. (5) that the tensor $M_{i j k l}$ forms the deviatoric part of the piezoresistance tensor under a coordinate transformation and has the following basic properties (see Appendix A):

$$
\begin{aligned}
& M_{1122}=M_{1212}, M_{2233}=M_{2323}, M_{3311}=M_{3131}, \\
& M_{1111}+M_{1133}+M_{1122}=M_{2211}+M_{2222}+M_{2233}=M_{3311}+M_{3322}+M_{3333}=1, \\
& M_{k k l l}=M_{k l k l}=3 .
\end{aligned}
$$

Table 1

Transformation matrix for Euler angles.

\begin{tabular}{cccl}
\hline & $x_{1}$ & $x_{2}$ & \multicolumn{1}{c}{$x_{3}$} \\
\hline$X_{1}$ & $\cos \phi \cos \theta \cos \psi$ & $\cos \phi \cos \theta \sin \psi$ & $-\cos \phi \sin \theta$ \\
& $-\sin \phi \sin \psi$ & $+\sin \phi \cos \psi$ & \\
$X_{2}$ & $-\sin \phi \cos \theta \cos \psi$ & $-\sin \phi \cos \theta \sin \psi$ & $\sin \phi \sin \theta$ \\
& $-\sin \phi \sin \psi$ & $+\cos \phi \cos \psi$ & \\
$X_{3}$ & $\sin \theta \cos \psi$ & $\sin \theta \sin \psi$ & $\cos \theta$ \\
\hline
\end{tabular}


We can now decompose the overall piezoresistance tensor into isotropic and deviatoric parts as (see Appendix B)

$$
\overline{\pi_{i j k l}}=\overline{\pi_{i j k l}^{0}}+\overline{\pi_{i j k l}^{\prime}},
$$

where $\overline{\pi_{i j k l}^{0}}$ and $\overline{\pi_{i j k l}^{\prime}}$ are the isotropic and deviatoric tensor components, respectively. According to Appendix B, $\overline{\pi_{k k l l}}=\overline{\pi_{k k l l}^{0}}$ and $\overline{\pi_{k l k l}}=\overline{\pi_{k l k l}^{0}}$ are the scalar invariants of the fourthrank tensor. Therefore, it can be immediately concluded that

$$
\overline{\pi_{k k l l}^{\prime}}=0, \overline{\pi_{k l k l}^{\prime}}=0
$$

The following physically significant properties for the deviatoric part of the overall piezoresistance tensor are given in Appendix C,

$$
\begin{aligned}
& \overline{\pi_{2323}^{\prime}}=\overline{\pi_{2233}^{\prime}}, \overline{\pi_{1313}^{\prime}}=\overline{\pi_{1133}^{\prime}}, \overline{\pi_{1212}^{\prime}}=\overline{\pi_{1122}^{\prime}}, \\
& \overline{\pi_{1111}^{\prime}}+\overline{\pi_{1313}^{\prime}}+\overline{\pi_{1212}^{\prime}}=0, \overline{\pi_{3333}^{\prime}}+\overline{\pi_{2323}^{\prime}}+\overline{\pi_{1313}^{\prime}}=0, \\
& \overline{\pi_{2222}^{\prime}}+\overline{\pi_{2323}^{\prime}}+\overline{\pi_{1212}^{\prime}}=0 .
\end{aligned}
$$

It can be concluded from Eq. (12) that the nine components of the deviatoric part of the overall piezoresistance tensor $\overline{\pi^{\prime}{ }_{i j k l}}$ are necessary to describe the anisotropy induced by the orthotropic texture and that the number of independent components of $\overline{\pi_{i j k l}^{\prime}}$ is only three.

The final form of the overall piezoresistance tensor equation is given in Appendix B,

$$
\begin{gathered}
\overline{\pi_{i j k l}^{0}} \equiv\left(\pi_{1}+\frac{1}{5} \pi_{3}\right) \delta_{i j} \delta_{k l}+2\left(\pi_{2}+\frac{1}{5} \pi_{3}\right) I_{i j k l}, \\
\overline{\pi_{i j k l}^{\prime}}=\pi_{3}\left[M_{i j k l}-\frac{1}{5}\left(\delta_{i j} \delta_{k l}+2 I_{i j k l}\right)\right] .
\end{gathered}
$$

The physical significance of Eq. (13) is that the hydrostatic stress component $\overline{\pi_{i j k l}^{0}}$ is invariant for both polycrystalline aggregates and a cubic single crystal, i.e., $\overline{\pi_{1111}^{0}}+\overline{\pi_{1122}^{0}}+\overline{\pi_{1133}^{0}}=\Pi_{1111}+2 \Pi_{1122}$. Another finding of physical significance is that there are only three independent components of $\overline{\pi_{i j k l}^{\prime}}$ for any choice of the CODF.

\subsection{Components of overall piezoresistance tensor}

The derived overall orthotropic piezoresistance tensor property is invariant for any choice of the functional form of the CODF. Therefore, we apply the CODF to the orthotropic texture 
defined by Roe in a sample calculation. ${ }^{(23)}$ As pointed out by Roe, the CODF can be expanded using the normalized generalized Legendre function $Z_{\alpha \beta \gamma}(\xi)$ as

$$
W(\xi, \psi, \phi)=\sum_{\alpha=0}^{\infty} \sum_{\beta=-\alpha \gamma=-\alpha}^{\alpha} \sum_{\alpha \beta \gamma}^{\alpha} Z_{\alpha \beta \gamma}(\xi) \exp (-i \beta \psi) \exp (-i \gamma \phi) .
$$

The tensor component of $A_{i j k l}(\xi, \psi, \phi)$ is expressed by three Euler angles. Thus, Eq. (9) can be expanded using the normalized generalized Legendre function $Z_{\alpha \beta \gamma}(\xi)$ as

$$
A_{i j k l}(\xi, \psi, \phi) \equiv \sum_{\alpha^{\prime}=0 \beta=-\alpha^{\prime} \gamma=-\alpha^{\prime}}^{\infty} \sum_{\alpha^{\prime} \beta \gamma}^{\alpha^{\prime}} \sum_{i j k l} Z_{\alpha^{\prime} \beta \gamma}(\xi) \exp (i \beta \psi) \exp (i \gamma \phi)
$$

where

$$
\left(a_{\alpha^{\prime} \beta \gamma}\right)_{i j k l}=\frac{1}{4 \pi^{2}} \int_{0}^{2 \pi 2 \pi} \int_{0}^{1} \int_{-1}^{1} A_{i j k l}(\xi, \psi, \phi) Z_{\alpha^{\prime} \beta \gamma}(\xi) \exp (-i \beta \psi) \exp (-i \gamma \phi) d \xi d \psi d \phi .
$$

The substitution of Eqs. (15) and (16) into Eq. (8) and the fundamental property for the integral of $\int_{-1}^{1} Z_{\alpha \beta \gamma}(\xi) Z_{\alpha^{\prime} \beta \gamma}(\xi) d \xi=\delta_{\alpha \alpha^{\prime}}$ lead to

$$
M_{i j k l}=4 \pi^{2} \sum_{\alpha=0}^{\infty} \sum_{\beta=-\alpha \gamma=-\alpha}^{\alpha} \sum_{\alpha \beta \gamma}^{\alpha}\left(a_{\alpha \beta \gamma}\right)_{i j k l} .
$$

Equation (18) can be reduced by using the rule of cubic crystal symmetry for the expansion coefficients derived by Roe (see Appendix D),

$$
M_{i j k l}=\frac{4 \pi^{2}}{7}\left[7 w_{000}\left(a_{000}\right)_{i j k l}+12 w_{400}\left(a_{400}\right)_{i j k l}+24 w_{420}\left(a_{420}\right)_{i j k l}+24 w_{440}\left(a_{440}\right)_{i j k l}\right],
$$

where $w_{000}=\frac{1}{4 \sqrt{2} \pi^{2}}$ and $\left(a_{000}\right)_{i j k l}=\frac{\sqrt{2}}{5}$.

The expressions for the expansion coefficients $a_{\alpha \beta \gamma}$ and three independent components of the tensor $M_{i j k l}$ are summarized in Tables 2 and 3, respectively. An example of calculation for $a_{\alpha \beta \gamma}$ and $M_{i j k l}$ is given in Appendix E.

The substitutions of $a_{\alpha \beta \gamma}$ and $M_{i j k l}$ in Tables 2 and 3 into Eqs. (11) and (14) give the longitudinal component of the overall piezoresistance tensor, 
Table 2

Examples of calculated $a_{\alpha \beta \gamma \gamma}$.

\begin{tabular}{cccc}
\hline & $i j k l=1122$ & $i j k l=2233$ & $i j k l=3311$ \\
\hline$\left(a_{000}\right)_{i j k l}$ & $\frac{\sqrt{2}}{5}$ & $\frac{\sqrt{2}}{5}$ & $\frac{\sqrt{2}}{5}$ \\
$\left(a_{400}\right)_{i j k l}$ & $\frac{\sqrt{2}}{60}$ & $-\frac{\sqrt{2}}{15}$ & $-\frac{\sqrt{2}}{15}$ \\
$\left(a_{420}\right)_{i j k l}$ & 0 & $-\frac{\sqrt{5}}{30}$ & $\frac{\sqrt{5}}{30}$ \\
$\left(a_{440}\right)_{i j k l}$ & $-\frac{\sqrt{35}}{60}$ & 0 & 0 \\
\hline
\end{tabular}

Table 3

Examples of calculated $M_{i j k l}$.

\begin{tabular}{lc}
\hline$i j k l=1122$ & $M_{i j k l}$ \\
\hline$i j k l=2233$ & $\frac{1}{5}+\frac{4 \sqrt{2} \pi^{2}}{35} w_{400}-\frac{8 \pi^{2}}{\sqrt{35}} w_{440}$ \\
$i j k l=3311$ & $\frac{16 \sqrt{2} \pi^{2}}{5}-\frac{16 \sqrt{2} \pi^{2}}{35} w_{400}-\frac{16 \sqrt{5} \pi^{2}}{35} w_{420}+\frac{16 \sqrt{5} \pi^{2}}{35} w_{420}$
\end{tabular}

$$
\overline{\pi_{1111}}=\left(\pi_{1}+2 \pi_{2}+\frac{3}{5} \pi_{3}\right)+\frac{\pi^{2}}{35}\left(12 \sqrt{2} w_{400}-16 \sqrt{5} w_{420}+8 \sqrt{5} w_{440}\right) \pi_{3}
$$

The remaining eight components can be easily derived from Eqs. (11) and (14) if necessary. In the special case of polycrystalline aggregates having the $<100>$ fiber texture, Eq. (20) can be reduced to (see Appendix E)

$$
\overline{\pi_{1111}}=\overline{\pi_{2222}}=\left(\pi_{1}+2 \pi_{2}+\frac{3}{5} \pi_{3}\right)+\frac{12 \sqrt{2} \pi^{2} w_{400 / 100}}{35} \pi_{3} .
$$

Thus, a single expansion coefficient $w_{400}$ (reduced to the normalized Legendre function) ${ }^{(25)}$ plays an important role in describing the strength of the fiber texture. In the case of isotropy, corresponding to a completely random distribution, $w_{400 / 100}=0$. On the other hand, in the case of an ideal fiber texture, in which all the crystal grain axes have the same specified fiber axis orientation, the expansion coefficient has a fixed value, i.e., $w_{400 / 100\rangle}=\frac{3}{4 \sqrt{2} \pi^{2}} \cong 0.0537$.

\section{Microfabrication and Experiment}

To verify the consistency between the proposed tensor equation and the experimental results, the microfabrication and texture and piezoresistance measurements of a poly-Si piezoresistor were carried out. The starting substrate was n-type (100) single-crystal silicon with a thermal$\mathrm{SiO}_{2}$ isolation layer of $0.7 \mu \mathrm{m}$ thickness. p-type poly-Si of $0.5 \mu \mathrm{m}$ thickness was deposited on the $\mathrm{SiO}_{2}$ isolation layer by low-pressure chemical vapor deposition (LPCVD). The reactor temperature and silane partial pressure during the LPCVD process were $873 \mathrm{~K}$ and $1 \mathrm{~Pa}$, respectively. The dopant concentration of the $\mathrm{p}$-type poly-Si was controlled by the implantation of boron ions. The crystal grain configuration was controlled by annealing with temperature up to $1373 \mathrm{~K}$ after the implantation. Samples with three different dopant concentrations were prepared: $N=10^{19} \mathrm{~cm}^{-3}$ (sheet resistance $\left.=1480 \Omega / \square\right), N=5 \times 10^{19} \mathrm{~cm}^{-3}$ (sheet resistance $=$ $139.5 \Omega / \square$ ), and $N=10^{20} \mathrm{~cm}^{-3}$ (sheet resistance $=75.1 \Omega / \square$ ). Average grain sizes with three different dopant concentrations were $0.13\left(N=10^{19} \mathrm{~cm}^{-3}\right), 0.12\left(N=5 \times 10^{19} \mathrm{~cm}^{-3}\right)$, and 0.12 
$\mu \mathrm{m}\left(N=10^{20} \mathrm{~cm}^{-3}\right)$. The average grain sizes obtained from the number average were in good agreement with the area average. Thus, it was confirmed that the p-type poly-Si is composed of single crystal grains.

Inverse pole figures of the p-type poly-Si thin films were obtained by the standard electron backscatter diffraction method using a system combining a field-emission scanning electron microscope (FE-SEM, JOEL: JSM-6500F) and an electron backscatter diffraction detector (EBSD, National Instruments: DVC 1412M).

Figure 2(a) shows the variation in the texture of poly-Si as a function of the temperature and silane pressure during the LPCVD process reported in Ref. 26. The poly-Si obtained in this work has a $<100>$ fiber texture as indicated by the tip of the red triangle in Fig. 2(a). Figure 2(b) shows typical inverse pole figures for the p-type poly-Si with the above dopant concentrations. The obtained inverse pole figures indicated that a strong $<100>$ fiber texture is dominant as pointed out by Wenk and coworkers. ${ }^{(26,28)}$ The relative intensity of the $<100>$ fiber texture was inversely proportional to the dopant concentration. The $<100>$ fiber texture approaches a random distribution for a relatively high dopant concentration.

p-type poly-Si piezoresistors and aluminum interconnections were fabricated by ultraviolet photolithography and reactive ion etching. Then, sintering was performed to form ohmic contacts between the p-type poly-Si piezoresistors and the aluminum interconnections. A fabricated poly-Si piezoresistor is shown in Fig. 3(a). The typical dimensions of the rectangular p-type poly-Si piezoresistor were a length of $80 \mu \mathrm{m}$ and a width of $8 \mu \mathrm{m}$. Finally, the n-type (100) single-crystal silicon substrate was cut into a rectangular microbeam and used to apply stress to the p-type poly-Si piezoresistors located on the substrate surface via a microbeam bending system, as described by Toriyama et al. ${ }^{(29)}$ The constitutive equation for the piezoresistance of polycrystalline aggregates in the material reference frame $O-x_{1} x_{2} x_{3}$ can be written as

$$
\frac{\Delta \rho_{i j}}{\rho_{0}}=\overline{\pi_{i j k l}} \sigma_{k l}
$$

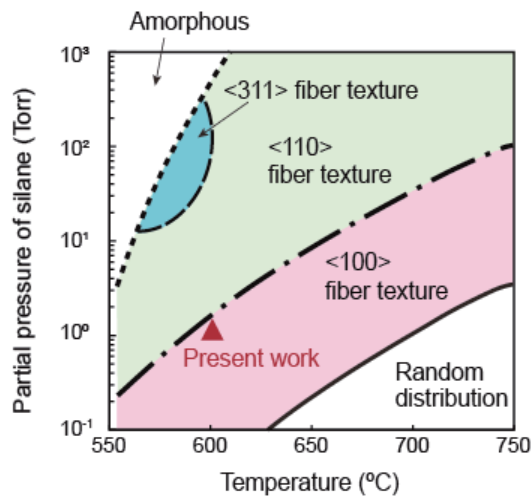

(a)

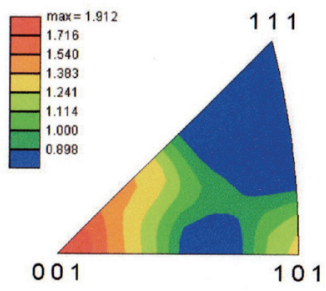

(b)

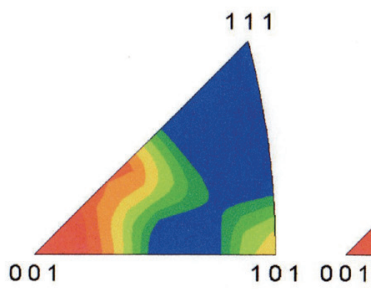

(c)

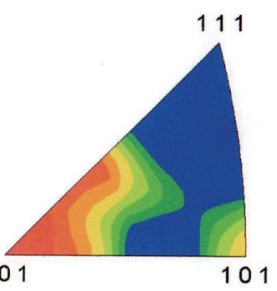

(d)

Fig. 2. (Color online) (a) Variation of the texture of poly-Si as a function of the temperature and silane pressure during the LPCVD process and (b-d) related pole figures. (b) $N=1 \times 10^{19} \mathrm{~cm}^{-3}$, (c) $N=5 \times 10^{19} \mathrm{~cm}^{-3}$, and (d) $N=$ $1 \times 10^{20} \mathrm{~cm}^{-3}$. 
where $\Delta \rho_{i j} / \rho_{0}$ and $\sigma_{k l}$ are the components of the relative changes in the resistivity and stress tensor, respectively. When the electrical field vector, current density vector, and in-plane $\left(x_{1} x_{2}\right.$ plane), uniaxial normal stress tensor are in the $x_{1}$-direction, the longitudinal component of the piezoresistance tensor can be determined from Eq. (22) as $\left(\Delta \rho_{11}\right) / \rho_{0}=\overline{\pi_{111}} \sigma_{11}$. Note that the $x_{3}$-direction of the fiber texture axis for the poly-Si piezoresistor coincides wth $<100>$, which is perpendicular to the n-type (100) single-crystal silicon substrate surface. The longitudinal component of the piezoresistance tensor has a transversely isotropic property and the $x_{1}$ direction can be selected as an arbitrary orientation in- $x_{1} x_{2}$ plane (see Appendix E). However, in the present work, we choose $<110>$ of the n-type (100) single-crystal silicon substrate as the $x_{1}$-direction of the poly-Si piezoresistor. Figure $3(\mathrm{~b})$ shows the relative change in the resistance of the p-type poly-Si piezoresistors as a function of applied stress. The change in resistance linearly increases with increasing applied stress for all samples with different carrier concentrations. It is clear that the variation in resistance with the applied stress increased as the carrier concentration decreased.

\section{Discussion}

On the basis of Eq. (21), the overall piezoresistance tensor component $\overline{\pi_{1111}}$ is composed of $\pi_{1}=\Pi_{1122}, \pi_{2}=\Pi_{2323}$, and $\pi_{1}+2 \pi_{1}+\pi_{3}=\Pi_{1111}$ related to the magnitude of piezoresistance components of the single-crystal silicon and the expansion coefficient $w_{400}$ related to the CODF. Thus, applying Eq. (21) to determine the experimental change in the expansion coefficient $w_{400}$ with the variation in crystal texture, the values of $\Pi_{1111}, \Pi_{1122}$, and $\Pi_{2323}$ for the p-type singlecrystal silicon must be specified. The values were obtained from the equation derived by Richter et al. ${ }^{(30)}$

$$
\begin{aligned}
& \Pi_{2323}\left(N_{A}, T\right)=C\left(N_{A}, T\right) \Pi_{2323, r e f}, \\
& \Pi_{1111}\left(N_{A}, T\right)=C\left(N_{A}, T\right) \Pi_{1111, r e f},
\end{aligned}
$$

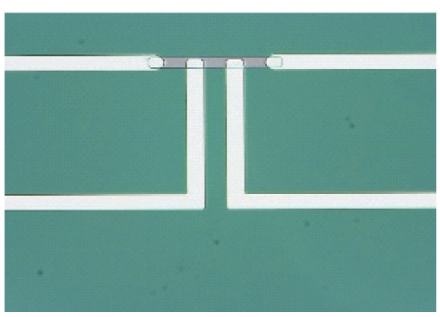

(a)

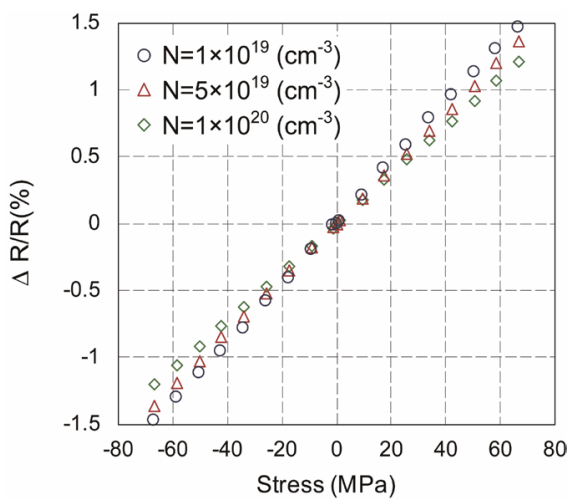

(b)

Fig. 3. (Color online) (a) Photograph of piezoresistor and (b) experimental results. 


$$
\begin{gathered}
\Pi_{1122}\left(N_{A}, T\right)=C\left(N_{A}, T\right) \Pi_{1122, \text { ref }}, \\
C\left(N_{A}, T\right) \equiv \frac{\Pi_{2323, \text { ref }}}{\left[1+\left(\frac{N_{A}}{6 \times 10^{19}}\right)^{0.43}\left(\frac{T}{300}\right)^{-0.1}+\left.\left(\frac{N_{A}}{7 \times 10^{20}}\right)^{1.6}\left(\frac{T}{300}\right)^{-3}\right|^{\left(\frac{T}{300}\right)^{-0.9}},\right.},
\end{gathered}
$$

where $N_{A}\left(\mathrm{~cm}^{-3}\right), T(\mathrm{~K})$, and $\Pi_{i j k l, r e f}$ are the carrier concentration, temperature, and reference piezoresistance coefficient $\Pi_{i j k l}$ at a very low carrier concentration $\left(N_{A}=10 \times 10^{14} \mathrm{~cm}^{-3}\right)$ at room temperature $(T=300 \mathrm{~K})$, respectively. Figure 4 shows a comparison of the experimental values of the present work and those partly taken from Ref. 31 and the theoretical prediction based on Richter's equation for $\Pi_{2323}, \Pi_{1111}$, and $\Pi_{1122}$. The validity of Richter's equation for the piezoresistance components of p-type single-crystal silicon can be confirmed from Fig. 4.

The preparation of $\pi_{1}, \pi_{2}$, and $\pi_{3}$ used for Eq. (21) has been completed. Then, the final task is to determine the experimental change in the expansion coefficient $w_{400}$ with the variation in crystal texture. The expansion coefficient $w_{400}$ can be determined by fitting the experimental data for $\overline{\pi_{1111}}$ to Eq. (21) after substituting the previously obtained values of $\pi_{1}, \pi_{2}$, and $\pi_{3}$. The obtained expansion coefficient $w_{400}$ values were 0.036 for $N=10^{19} \mathrm{~cm}^{-3}, 0.026$ for $N=5 \times 10^{19} \mathrm{~cm}^{-3}$, and 0.012 for $N=10^{20} \mathrm{~cm}^{-3}$. Figure 5 shows the relationship between the overall piezoresistance tensor component $\overline{\pi_{1111}}$ and the expansion coefficient $w_{400}$ of the CODF obtained from Eq. (21).

On the basis of Eq. (21), the magnitude of the overall piezoresistance tensor component $\overline{\pi_{1111}}$ may proportionally correlate with that of the expansion coefficient $w_{400}$ at a constant dopant concentration, i.e., $\pi_{1}, \pi_{2}$, and $\pi_{3}$ are constant. The variation in the magnitude of $w_{400}$ correlated with the growth of fiber texture and that of random distribution. The $<100>$ fiber texture tended to show a random distribution with increasing dopant concentration, as also

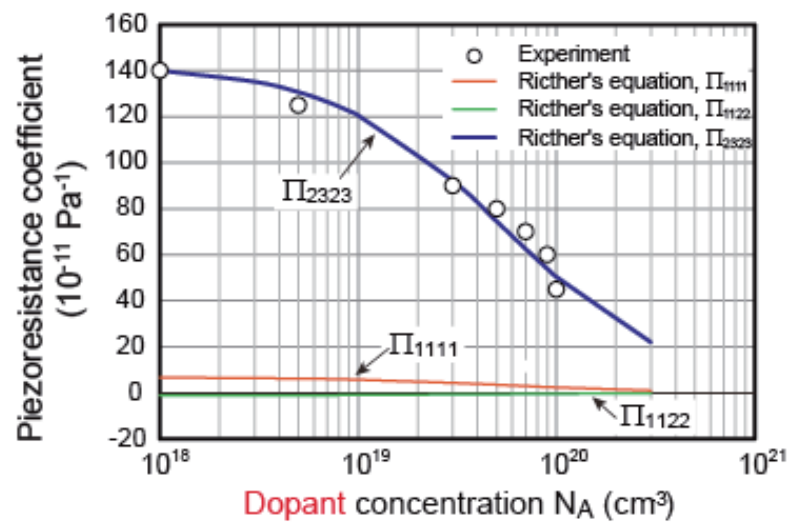

Fig. 4. (Color online) Comparison of experimental values in Ref. 31 and the theoretical prediction based on Richter's equation for $\Pi_{2323}$.

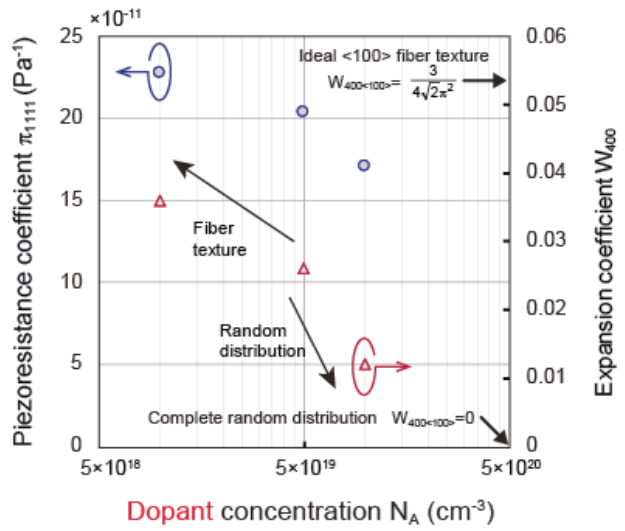

Fig. 5. (Color online) Comparison between analytical and experimental results for piezoresistance coefficient $\pi_{1111}$. 
revealed by the results of pole figure measurement in Figs. 2(b) and 5. The magnitude of the overall piezoresistance tensor component $\overline{\pi_{1111}}$ also tended to decrease with increasing dopant concentration, as revealed by the results in Fig. 5. Equation (21) shows the experimental evidence indicating that both magnitudes of the overall piezoresistance tensor component $\overline{\pi_{1111}}$ and expansion coefficient $w_{400}$ increased with the growth of $<100>$ fiber texture. On the other hand, both of $\overline{\pi_{1111}}$ and $w_{400}$ decreased with the growth of random distribution.

Therefore, we can conclude that the proposed overall piezoresistance tensor equation with a spherical harmonics expansion is expected to be useful for determining the role of the preferential crystallographic orientation in the piezoresistance anisotropy of polycrystalline aggregates.

\section{Conclusions}

We have derived the overall piezoresistance tensor for polycrystalline aggregates having an orthotropic texture in terms of the CODF and the cubic single-crystal piezoresistance tensor. It was shown that the overall piezoresistance tensor for polycrystalline aggregates has nine components and can be decomposed into isotropic and deviatoric parts. We found that the nine deviatoric tensor components are functions of the preferential crystallographic orientation of the cubic single crystals and that the number of independent components is greatly reduced to three.

To verify the consistency between the derived overall piezoresistance tensor and experimental results, the microfabrication and texture and piezoresistance measurements of a poly-Si piezoresistor were carried out. It can be demonstrated that the proposed overall piezoresistance tensor equation with a spherical harmonics expansion is expected to be useful for determining the role of the preferential crystallographic orientation in the piezoresistance anisotropy of polycrystalline aggregates.

\section{Acknowledgments}

This work was supported by JSPS KAKENHI Grant Number JP16H03819.

\section{References}

1 A. A. Barlian, W. T. Park, J. R. Mallon, Jr., A. J. Rastegar, and B. L. Pruitt: Proc. IEEE 97 (2009) 513.

2 V. Mosser, J. Suski, and J. Goss: Sens. Actuators, A 28 (1991) 113.

3 M. Tortonese, R. C. Barrett, and C. F. Quate: Appl. Phys. Lett. 62 (1993) 834.

4 W. P. Eaton and J. H. Smith: Smart Mater. Struct. 6 (1997) 530.

5 J. Bartholomeczik, P. Ruther, and O. Paul: IEEE Sens. J. 5 (2005) 872.

6 P. J. French and A. G. R. Evans: J. Phys. E: Sci. Instrum. 19 (1986) 1055.

7 P. J. French and A. G. R. Evans: Solid State Electron. 32 (1989) 1.

8 P. J. French: Sens. Actuators, A 99 (2002) 3.

9 T. I. Kamins: Sens. Actuators, A 21-23 (1990) 817.

10 M. L. Berre, M. Lemiti, D. Barbier, P. Pinard, J. Cali, E. Bustarret, J. Sicart, and J. L. Robert: Sens. Actuators, A 46-47 (1995) 166. 
11 V. A. Gridchin, V. M. Lubimsky, and M. P. Sarina: Sens. Actuators, A 49 (1995) 67.

12 M. L. Berre, P. Kleimann, B. Semmache, D. Barbier, and P. Pinard: Sens. Actuators, A 54 (1996) 700.

13 T. L. Waterfall, G. K. Johns, R. K. Messenger, B. D. Jensen, T. W. Mclain, and L. L. Howell: Sens. Actuators, A 141 (2008) 610.

14 S. K. Patil, Z. C.-Butler, and D. P. Butler: Thin Solid Films 519 (2010) 479.

15 S. Uhlig, S. Rau, and G. Schultes: Sens. Actuators, A 172 (2011) 447.

16 P. O. Renault, E. Le Bourhis, P. Villain, Ph. Goudeau, K. F. Badawi, and D. Faurie: Appl. Phys. Lett. 83 (2003) 473.

17 T. Ando and T. Toriyama: IEEJ Trans. SM 138 (2018) 214 (in Japanese).

18 H. J. Bunge: Texture Analysis in Materials Science (Butterworth, 1982) p. 42.

19 A. Ruess and Z. Angew: Math. Mech. 9 (1929) 49.

20 T. Toriyama, Y. Yokoyama, and S. Sugiyama: Sens. Mater. 124 (2000) 73.

21 N. Matsuzuka and T. Toriyama: J. Appl. Phys. 108 (2010) 064902.

22 H. Margenau and G. M. Murphy: The Mathematics of Physics and Chemistry (Young Press, 2009) 2nd ed., p. 289.

23 R. J. Roe: J. Appl. Phys. 36 (1965) 2024.

24 R. J. Roe: J. Appl. Phys. 37 (1966) 2069.

25 E. T. Whittaker and G. N. Watson: A Course of Modern Analysis (Cambridge University Press, 1927) 4th ed., p. 323.

26 P. Joubert, B. Loisel, Y. Chouan, and L. Haji: J. Electrochem. Soc. 134 (1987) 2541.

27 H. R. Wenk, J. Huang, G. C. Johnson, and R. T. Howe: J. Appl. Phys. 67 (1990) 572.

28 H. R. Wenk and P. Van Houtte: Rep. Prog. Phys. 67 (2004) 1367.

29 T. Toriyama, D. Funai, and S. Sugiyama: J. Appl. Phys. 93 (2003) 561.

30 J. Richter, J. Pedersen, M. Brandbyge, E. V. Thomson, and O. Hansen: J. Appl. Phys. 104 (2008) 023715.

31 T. Toriyama and S. Sugiyama: J. Microelectromech. Syst. 11 (2002) 598.

32 P. Kleimann, B. Semmache, M. Le Berre, and D. Barbier: Phys. Rev. B, 57 (1998) 8966.

33 H. Jeffreys and B. Jeffreys: Methods of Mathematical Physics (Cambridge University Press, 1972) 3rd ed., p. 88.

\section{Appendix}

\section{A. Derivation of basic properties of tensor $M_{i j k l}$}

We can find a symmetrical property of $A_{i j k l}(\xi, \psi, \phi)$ from Eq. (9) as

$$
A_{\alpha \alpha \beta \beta}(\xi, \psi, \phi)=\sum_{L=1}^{3} \frac{\partial X_{L}}{\partial x_{\alpha}} \frac{\partial X_{L}}{\partial x_{\alpha}} \frac{\partial X_{L}}{\partial x_{\beta}} \frac{\partial X_{L}}{\partial x_{\beta}}=\sum_{L=1}^{3} \frac{\partial X_{L}}{\partial x_{\alpha}} \frac{\partial X_{L}}{\partial x_{\beta}} \frac{\partial X_{L}}{\partial x_{\alpha}} \frac{\partial X_{L}}{\partial x_{\beta}}=A_{\alpha \beta \beta \beta}(\xi, \psi, \phi) .
$$

Then, a symmetrical property of $M_{i j k l}(\xi, \psi, \phi)$ defined by Eq. (8) is

$$
\begin{aligned}
M_{\alpha \alpha \beta \beta} & =\int_{0}^{2 \pi} \int_{0}^{2 \pi} \int_{-1}^{1} W(\xi, \psi, \phi) A_{\alpha \alpha \beta \beta}(\xi, \psi, \phi) d \xi d \psi d \phi, \\
& =\int_{0}^{2 \pi} \int_{0}^{2 \pi} \int_{-1}^{1} W(\xi, \psi, \phi) A_{\alpha \beta \alpha \beta}(\xi, \psi, \phi) d \xi d \psi d \phi=M_{\alpha \beta \alpha \beta} .
\end{aligned}
$$

A similar cyclic permutation derives the symmetrical properties of $M_{i j k l}(\xi, \psi, \phi)$, 


$$
M_{\alpha \alpha \beta \beta}=M_{\alpha \beta \alpha \beta}=M_{\beta \alpha \beta \alpha}=M_{\beta \alpha \alpha \beta}=M_{\alpha \beta \beta \alpha}=M_{\beta \beta \alpha \alpha},
$$

where the summation convention does not apply to Greek indices (e.g., $\alpha, \beta)$.

We can find a normalized property of $A_{i j k l}(\xi, \psi, \phi)$ from Eq. (9) as

$$
\begin{gathered}
A_{1111}(\xi, \psi, \phi)+A_{3131}(\xi, \psi, \phi)+A_{1212}(\xi, \psi, \phi) \\
=\sum_{L=1}^{3}\left(\frac{\partial X_{L}}{\partial x_{1}}\right)^{4}+\sum_{L=1}^{3}\left(\frac{\partial X_{L}}{\partial x_{3}}\right)^{2}\left(\frac{\partial X_{L}}{\partial x_{1}}\right)^{2}+\sum_{L=1}^{3}\left(\frac{\partial X_{L}}{\partial x_{1}}\right)^{2}\left(\frac{\partial X_{L}}{\partial x_{2}}\right)^{2} \\
=\sum_{L=1}^{3}\left(\frac{\partial X_{L}}{\partial x_{1}}\right)^{2}\left[\left(\frac{\partial X_{L}}{\partial x_{1}}\right)^{2}+\left(\frac{\partial X_{L}}{\partial x_{3}}\right)^{2}+\left(\frac{\partial X_{L}}{\partial x_{2}}\right)^{2}\right]=\sum_{L=1}^{3}\left(\frac{\partial X_{L}}{\partial x_{1}}\right)^{2}=1 .
\end{gathered}
$$

Then, a normalized property of $M_{i j k l}(\xi, \psi, \phi)$ defined by Eq. (8) is

$$
\begin{aligned}
M_{1111}+ & M_{3131}+M_{1212} \\
& =\int_{0}^{2 \pi 2 \pi} \int_{0}^{1} W(\xi, \psi, \phi)\left[A_{1111}(\xi, \psi, \phi)+A_{3131}(\xi, \psi, \phi)+A_{1212}(\xi, \psi, \phi)\right] d \xi d \psi d \phi \\
& =\int_{0}^{2 \pi 2 \pi} \int_{0}^{1} \int_{-1}^{1} W(\xi, \psi, \phi) d \xi d \psi d \phi=M_{1111}+M_{1133}+M_{1122}=1 .
\end{aligned}
$$

A similar cyclic permutation derives the normalized properties of $M_{i j k l}(\xi, \psi, \phi)$,

$$
M_{2211}+M_{2222}+M_{2233}=M_{3311}+M_{3322}+M_{3333}=1 .
$$

A combination of Eqs. (A5) and (A6) leads to

$$
\begin{aligned}
& M_{k k l l}=M_{1111}+M_{1122}+M_{1133}+M_{2211}+M_{2222}+M_{2233}+M_{3311}+M_{3322}+M_{3333}=3, \\
& M_{k l k l}=M_{1111}+M_{1212}+M_{1313}+M_{2121}+M_{2222}+M_{2323}+M_{3131}+M_{3232}+M_{3333}=3 .
\end{aligned}
$$

\section{B. Isotropic and deviatoric fourth-rank tensors}

Components of the isotropic tensor are invariant with respect to the coordinate transformation. We can make an isotropic fourth-rank tensor from an arbitrary fourth-rank tensor with the symmetry of $\overline{\pi_{i j k l}}=\overline{\pi_{j i k l}}=\overline{\pi_{i j l k}}$, ${ }^{(32)}$ 


$$
\overline{\pi_{i j k l}^{0}}=P_{1} \delta_{i j} \delta_{k l}+2 P_{2} I_{i j k l},
$$

where

$$
\begin{aligned}
& P_{1}=\frac{1}{15}\left(2 \overline{\pi_{p p q q}}-\overline{\pi_{p q p q}}\right), \\
& P_{2}=\frac{1}{30}\left(\overline{3 \pi_{p q p q}}-\overline{\pi_{p p q q}}\right) .
\end{aligned}
$$

A deviatoric part of the tensor can be obtained by subtracting the isotropic part from the original tensor,

$$
\overline{\pi_{i j k l}^{\prime}}=\overline{\pi_{i j k l}}-\overline{\pi_{i j k l}^{0}} \text {. }
$$

Substituting Eq. (7), i.e., $\overline{\pi_{i j k l}}=\pi_{1} \delta_{i j} \delta_{k l}+2 \pi_{2} I_{i j k l}+\pi_{3} M_{i j k l}$ into Eqs. (B2) and (B3), we obtain

$$
\begin{aligned}
& \overline{\pi_{p p q q}}=\pi_{1} \delta_{p p} \delta_{q q}+2 \pi_{2} I_{p p q q}+\pi_{3} M_{p p q q}=9 \pi_{1}+6 \pi_{2}+3 \pi_{3}, \\
& \overline{\pi_{p q p q}}=\pi_{1} \delta_{p q} \delta_{p q}+2 \pi_{2} I_{p q p q}+\pi_{3} M_{p q p q}=3 \pi_{1}+12 \pi_{2}+3 \pi_{3}, \\
& P_{1}=\pi_{1}+\frac{1}{5} \pi_{3}, \text { and } P_{2}=\pi_{2}+\frac{1}{5} \pi_{3} .
\end{aligned}
$$

Thus, Eqs. (B1) and (B4) can be rewritten by using Eqs. (B2), (B3), (B5), (B6), and (B7),

$$
\begin{gathered}
\overline{\pi_{i j k l}^{0}}=\left(\pi_{1}+\frac{1}{5} \pi_{3}\right) \delta_{i j} \delta_{k l}+2\left(\pi_{2}+\frac{1}{5} \pi_{3}\right) I_{i j k l}, \\
\overline{\pi_{i j k l}^{\prime}}=\pi_{3}\left[M_{i j k l}-\frac{1}{5}\left(\delta_{i j} \delta_{k l}+2 I_{i j k l}\right)\right] .
\end{gathered}
$$

\section{Derivation of basic properties of deviatoric tensor $\left(\overline{\pi_{i j k l}}\right)_{\text {deviatoric }}$}

A combination of Eq. (14), i.e., $\overline{\pi_{i j k l}^{\prime}}=\pi_{3}\left[M_{i j k l}-\frac{1}{5}\left(\delta_{i j} \delta_{k l}+2 I_{i j k l}\right)\right]$, and Eq. (8) lead to basic properties of the deviatoric tensor as follows:

$$
\left(\overline{\pi_{k k l l}}\right)_{\text {deviatoric }} \overline{\pi_{k k l l}^{\prime}}=\pi_{3}\left[M_{k k l l}-\frac{1}{5}\left(\delta_{k k} \delta_{l l}+2 I_{k k l l}\right)\right]=\pi_{3}(3-3)=0,
$$




$$
\begin{gathered}
\left(\overline{\pi_{k l k l}}\right)_{\text {deviatoric }}=\pi_{3}\left[M_{k l k l}-\frac{1}{5}\left(\delta_{k l} \delta_{k l}+2 I_{k l k l}\right)\right]=\pi_{3}(3-3)=0 . \\
\left(\overline{\pi_{2323}}\right)_{\text {deviatoric }}=\pi_{3}\left[M_{2323}-\frac{1}{5}\left(\delta_{23} \delta_{23}+2 I_{2323}\right)\right]=\pi_{3}\left(M_{2323}-\frac{1}{5}\right), \\
\left(\overline{\pi_{2233}}\right)_{\text {deviatoric }}=\pi_{3}\left[M_{2233}-\frac{1}{5}\left(\delta_{22} \delta_{33}+2 I_{2233}\right)\right]=\pi_{3}\left(M_{2233}-\frac{1}{5}\right) .
\end{gathered}
$$

However, when $M_{2323}=M_{2233}$ from Eq. (A3),

$$
\begin{gathered}
\left(\overline{\pi_{2323}}\right)_{\text {deviatoric }}=\left(\overline{\pi_{2233}}\right)_{\text {deviatoric }} . \\
\left(\overline{\pi_{1111}}\right)_{\text {deviatoric }}=\pi_{3}\left[M_{1111}-\frac{1}{5}\left(\delta_{11} \delta_{11}+2 I_{1111}\right)\right]=\pi_{3}\left(M_{1111}-\frac{3}{5}\right), \\
\left(\overline{\pi_{1313}}\right)_{\text {deviatoric }}=\pi_{3}\left[M_{1313}-\frac{1}{5}\left(\delta_{13} \delta_{13}+2 I_{1313}\right)\right]=\pi_{3}\left(M_{1313}-\frac{1}{5}\right), \\
\left(\overline{\pi_{1212}}\right)_{\text {deviatoric }}=\pi_{3}\left[M_{1212}-\frac{1}{5}\left(\delta_{12} \delta_{12}+2 I_{1212}\right)\right]=\pi_{3}\left(M_{1212}-\frac{1}{5}\right) .
\end{gathered}
$$

However, when $M_{1111}+M_{1313}+M_{1212}=1$ from Eq. (A6),

$$
\left(\overline{\pi_{1111}}\right)_{\text {deviatoric }}+\left(\overline{\pi_{1313}}\right)_{\text {deviatoric }}+\left(\overline{\pi_{1212}}\right)_{\text {deviatoric }}=0 .
$$

\section{Rule of cubic crystal symmetry for the expansion coefficients defined by Roe}

It can be estimated from Eqs. (8) and (9) and Table 1 that the component of $A_{i j k l}(\xi, \psi, \phi)$ is a fourth-order polynominal in terms of the trigonometric function of Euler angles. Therefore, $\beta$ and $\gamma$ in the exponents of Eq. (16) take the ranges of $-4 \leq \beta, \gamma \leq 4$. The expansion coefficients between $\left(a_{000}\right)_{i j k l}$ and $\left(a_{444}\right)_{i j k l}$ may be shown in Eq. (16). The rule of cubic crystal symmetry for the expansion coefficients $\left(a_{l m n}\right)_{i j k l}$ may be summarized as follows: ${ }^{(23,24)}$

$$
\begin{gathered}
\left(a_{l m n}\right)_{i j k l} \neq 0(n=\text { even }),\left(a_{l m n}\right)_{i j k l}=0(n=\text { odd }), \\
\left(a_{l m n}\right)_{i j k l}=\left(a_{l m(-n)}\right)_{i j k l},\left(a_{2 m 0}\right)_{i j k l}=0, \text { and }\left(a_{4 m 4}\right)_{i j k l}=\frac{5}{\sqrt{70}}\left(a_{4 m 0}\right)_{i j k l} .
\end{gathered}
$$

Substituting Roe's symmetrical properties into Eq. (18), we can find that the four-independent combinations of $w_{000}\left(a_{000}\right)_{i j k l}, w_{400}\left(a_{400}\right)_{i j k l}, w_{420}\left(a_{420}\right)_{i j k l}$, and $w_{440}\left(a_{440}\right)_{i j k l}$ may be shown in Eq. (19), and that other combinations vanish. 


\section{E. Derivation procedure for component of $\overline{\pi_{i j k l}}$}

The longitudinal and transverse components of the overall piezoresistance tensor $\overline{\pi_{1111}}$ [Eq. (21)] and $\overline{\pi_{1122}}$ are derived as an example. The component of $a_{1122}(\xi, \psi, \phi)$ can be derived from the combination of Eq. (9) and Table 1,

$$
A_{1122}(\xi, \psi, \phi)=\sum_{L=1}^{3}\left(\frac{\partial X_{L}}{\partial x_{1}}\right)^{2}\left(\frac{\partial X_{L}}{\partial x_{2}}\right)^{2}
$$

The substitution of Eq. (A1) into Eq. (17) leads to

$$
\begin{aligned}
\left(a_{400}\right)_{1122} & =\frac{1}{4 \pi^{2}} \int_{0}^{2 \pi 2 \pi} \int_{0}^{1} \int_{-1}^{1} A_{1122}(\xi, \psi, \phi) Z_{400}(\xi) \exp (-i 0 \psi) \exp (-i 0 \phi) d \xi d \psi d \phi \\
& =\frac{1}{32} \int_{-1}^{1}\left(7 \xi^{4}-6 \xi^{2}+7\right) Z_{400}(\xi) d \xi \\
& =\frac{1}{32} \int_{-1}^{1}\left(\frac{8}{5} P_{4}^{0}(\xi)+\frac{32}{5} P_{0}^{0}(\xi)\right) \frac{3}{\sqrt{2}} P_{4}^{0}(\xi) d \xi=\frac{\sqrt{2}}{60},
\end{aligned}
$$

where

$$
Z_{n m 0} \equiv \sqrt{\left(\frac{2 n+1}{2}\right) \frac{(n-m) !}{(n+m) !}} P_{n}^{m}(\xi)
$$

is the associated Legendre function with the orthogonal property, ${ }^{(25)}$

$$
\begin{gathered}
\int_{-1}^{1} P_{n}^{m}(\xi) P_{l}^{m}(\xi) d \xi=0 \quad(n=l), \\
\int_{-1}^{1} P_{n}^{m}(\xi) P_{n}^{m}(\xi) d \xi=\left(\frac{2}{2 n+1}\right) \frac{(n+m) !}{(n-m) !} \quad(n \neq l) .
\end{gathered}
$$

A similar calculation derives another component of $\left(a_{000}\right)_{1122}=\frac{\sqrt{2}}{5}$ and $\left(a_{440}\right)_{1122}=-\frac{\sqrt{35}}{60}$. Note that $\left(a_{420}\right)_{1122}=0$, because only fourth-order terms of $\exp ( \pm i 4 \psi)$ are shown in Eq. (E1) (see Table 2).

Therefore, the component of Eq. (19) associated with $A_{1122}(\xi, \psi, \phi)$ is (see Table 3 ) 


$$
M_{1122}=\frac{1}{5}+\frac{4 \sqrt{2} \pi^{2}}{35} w_{400}-\frac{8 \pi^{2}}{\sqrt{35}} w_{440}
$$

A similar calculation derives another component associated with $A_{3311}(\xi, \psi, \phi)$

$$
M_{3311}=\frac{1}{5}-\frac{16 \sqrt{2} \pi^{2}}{35} w_{400}+\frac{16 \sqrt{5} \pi^{2}}{35} w_{440} .
$$

Finally, the substitution of Eqs. (E4) and (E5) with the relation $M_{1111}=1-\left(M_{1122}+M_{3311}\right)$ [Eq. (10)] into Eqs. (11) and (14) leads to

$$
\overline{\pi_{1111}}=\left(\pi_{1}+2 \pi_{2}+\frac{3}{5} \pi_{3}\right)+\frac{\pi^{2}}{35}\left(12 \sqrt{2} w_{400}-16 \sqrt{5} w_{420}+8 \sqrt{5} w_{440}\right) \pi_{3} .
$$

Equation (19) associated with $A_{2233}(\xi, \psi, \phi)$ derives

$$
M_{2233}=\frac{1}{5}-\frac{4 \sqrt{2} \pi^{2}}{35} w_{400}-\frac{8 \pi^{2}}{\sqrt{35}} w_{440}
$$

Using the relation $M_{2222}=1-\left(M_{1122}+M_{2233}\right)$, Eqs. (E4), (E7), (11), and (14) lead to

$$
\overline{\pi_{2222}}=\left(\pi_{1}+2 \pi_{2}+\frac{3}{5} \pi_{3}\right)+\frac{\pi^{2}}{35}\left(12 \sqrt{2} w_{400}+16 \sqrt{5} w_{420}+8 \sqrt{5} w_{440}\right) \pi_{3} .
$$

Equation (19) associated with $A_{1122}(\xi, \psi, \phi)$ also derives

$$
\overline{\pi_{1122}}=\left(\pi_{1}+\frac{1}{5} \pi_{3}\right)+\frac{4 \sqrt{2} \pi^{2}}{35}\left(w_{400}-\sqrt{70} w_{440}\right) \pi_{3} .
$$

In the case of fiber texture, we can choose $w_{420}=w_{440}=0$. Therefore, we can derive from Eqs. (E6) and (E8) that the longitudinal piezoresistance tensor component is invariant with respect to the coordinate rotation around the axis of fiber texture, i.e., has a transversely isotopic property,

$$
\overline{\pi_{1111}}=\overline{\pi_{2222}}=\left(\pi_{1}+2 \pi_{2}+\frac{3}{5} \pi_{3}\right)+\frac{12 \sqrt{2} \pi^{2}}{35} w_{400} \pi_{3} .
$$




\section{About the Authors}

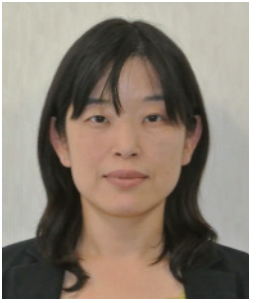

Taeko Ando received her B.E. degree in aerospace engineering and her M.S. and Ph.D. degrees in microsystem engineering from Nagoya University, Japan, in 1996, 1999, and 2001, respectively. From 2001 to 2008, she was an assistant professor and, from 2008 to 2009, a senior lecturer at Nagoya University, Japan. Since 2009, she has been an associate professor at Ritsumeikan University. Her research interests are in the physical properties of MEMS material in a micro/nanoscale.

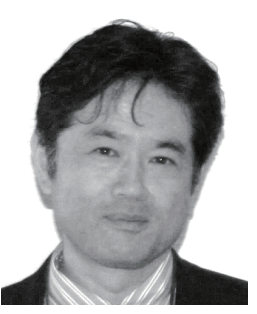

Toshiyuki Toriyama received his Ph.D. degree from Kyushu University, Japan, in 1994. Since 2004, he has been a professor at Ritsumeikan University, Japan. His research interests are in piezoresistance materials and microscale turbomachinery. 